\title{
Hospital Competition and Unplanned Readmission: Evidence from a Systematic Review
}

This article was published in the following Dove Press journal:

Risk Management and Healthcare Policy

\author{
Qingling Jiang ${ }^{1,2}$ \\ Fan Tian ${ }^{1,2}$ \\ Zhenmi Liu ${ }^{2,3}$ \\ Jay $\operatorname{Pan} \mathbb{D}^{1,2}$
}

'Department of Epidemiology and Health Statistics, West China School of Public Health and West China Fourth Hospital, Sichuan University, Chengdu, Sichuan Province, People's Republic of China; ${ }^{2}$ Institute for Healthy Cities and West China Research Center for Rural Health Development, Sichuan University, Chengdu, Sichuan Province, People's Republic of China; ${ }^{3}$ Department of Maternal, Child and Adolescent Health, West China School of Public Health and West China Fourth Hospital, Sichuan University, Chengdu, Sichuan Province, People's Republic of China
Correspondence: Zhenmi Liu; Jay Pan Email zhenmiliu@scu.edu.cn; panjie. jay@scu.edu.cn

\begin{abstract}
Competition has been widely introduced among hospitals in the hope of improving health-care quality. However, whether competition leads to higher-quality health care is a topic of considerable debate. We conducted a systematic review to assess the impact of hospital-market competition on unplanned readmission. We searched six electronic databases (PubMed, EmBase, Wiley Online Library, Web of Science, Scopus, and JSTOR) and reference lists of screened articles for relevant studies, and strictly followed methods proposed by the Cochrane Collaboration. Finally, nine observational studies with 2,241,767 patients were included. For the primary outcome, pooled results of three studies showed that it was uncertain whether or not hospital competition reduces readmission ( $\beta=0.02, \mathrm{P}=0.06$; very low certainty of evidence, as they were all observational studies with high heterogeneity). Inconsistent results were found in the remaining six studies, and they were assessed as very low-certainty evidence, downgraded for either inconsistency or indirectness or both. As for secondary outcomes, seven of the nine studies reported on the impact of competition on the risk of mortality, and two reported on length of stay (LOS). It was uncertain whether competition had an effect on mortality or LOS. The relevant studies were limited and of very low certainty, which means there is currently no reliable evidence showing that hospital competition reduces quality of health care in terms of readmission/ mortality/LOS. There is a need for rigorous studies to assess the impact of hospital competition on the quality of health care.
\end{abstract}

Keywords: hospital market, competition, readmission, quality of care, health policy

\section{Background}

It is often argued that encouraging competition among health-care providers will improve quality. Many countries have implemented procompetition policies among hospitals, such as China, who carried out market-oriented health-care reform in $1985,{ }^{1}$ the US, whose delivery system is private market-based, and the UK, where patients have been allowed to choose any qualified provider since $2008,2,3$ encouraging hospitals to compete for patients on quality. Under classical economic theory, competition effectively mobilizes production, as commercial firms relentlessly pursue lower costs and new products and technologies to attract consumers. ${ }^{4}$ Therefore, competition generally brings a higher quality of products and services from both the supply side and the customers' side $^{5,6}$ of the market. This theory is believed to hold true for most industries. ${ }^{4,7}$ However, when applying this assumption to the organization of health care, whether market competition is an efficient approach to promote quality of care has been a subject of debate for a long time. Indeed, the premises of classical economic theory in predicting the effectiveness of competition may not 
obtain in health-care markets. ${ }^{8,9}$ For instance, one may receive differentiated health care from any given hospital. ${ }^{9}$ There is serious information asymmetry in that suppliers have more knowledge of diseases and treatments than patients, which can lead to induced demand. Whether health care-market competition positively influences hospital service quality is controversial. ${ }^{1,10}$ As such, we aimed to assess the impact of hospital-market competition on quality of care.

\section{Intervention - Hospital Competition}

Health-care competition exists at three levels: individuals who provide health care (eg, health-care practitioners), organizations that provide health-care services (eg, hospitals or health-service centers), and organizations that provide health-care financing, insurance, and health-care plans (eg, insurance companies). In this review, we focus on competition at the level of health care-service providers.

When measuring competition at the level of health care-service providers, two measures are widely used: number of hospitals (NoH) and the HerfindahlHirschman Index (HHI). ${ }^{11,12}$ The HHI is the sum of squared market shares for all hospitals in the market. Its reliability has been questioned, as an endogeneity problem may arise due to hospitals with higher quality attracting more patients, with their own higher market share then resulting in a higher HHI value. In this case, it is difficult to determine the impact of competition on quality, due to the problem of reverse causality. ${ }^{13}$ However, it is still the most popular measurement for hospital competition in empirical work, due to its good calculability, no requirement for a preferred alternative measure, and representability of both $\mathrm{NoH}$ and market share. Therefore, we included only studies using the HHI as a competition indicator in this review.

Appropriately defining the hospital market is a prerequisite for capturing the actual situation of hospital competition. The definition of hospital market area used when calculating HHI can vary from study to study with different advantages/ disadvantages, which affects HHI values. ${ }^{14,15}$ Rather than exploring the competition status of the hospital market, the aim of this review was to explore the relationship between hospital-service quality and hospital competition, and thus no matter how authors defined the hospital market area, HHI can still be a proper variable for competition. In this way, this review accepted all authors' definitions of hospital market.

\section{Concepts of Health-Care Quality}

Experts have struggled to formulate a generally applicable definition of the quality of health care for a long time. $^{16}$

In order to choose a quality indicator to evaluate care quality for external purposes, certain criteria need to be fulfilled in regard to reliability and validity. An indicator needs to show relevance, based on its impact on health, its importance for policy, and its susceptibility to being influenced by the health-care system. Readmissions, especially unplanned ones, can normally be avoided with appropriate in-hospital care, but cause a high burden to health-care systems and patients, ${ }^{17,18}$ hence unplanned-readmission rate is commonly used for measuring quality of care delivered during the hospital stay and is used in this review too. ${ }^{19-22}$ It is worth mentioning that there are two assumptions when associating unplanned readmissions with quality: hospitals with more patients who are sicker will have higher readmission rates, and the conditions treated at hospitals are similar in distribution. Readmission can be interpreted as a measure of quality only if one controls for these two things.

Readmissions cannot happen when patients die during their hospital stay, which is captured by the mortality rate. As such, mortality can be seen as an end point competing with readmissions, and hospital deaths should be subtracted from total patients to calculate the readmission rate. Also, in settings where hospital readmission-reduction programs are emphasized, mortality rates seem to have increased. ${ }^{23}$ Therefore, mortality rates should be borne in mind alongside readmission rates to gain insight in hospital performance.

Length of stay (LOS) is another outcome measure associated with readmission, though their relationship is elusive. ${ }^{24-26}$ Longer LOS means more clinical care and lower bed-turnover ratio, which incurs high medical costs and reduction in hospital efficiency. ${ }^{27}$ As such, some hospitals try to shorten LOS for patients, which may cause higher readmission rates.

In summary, unplanned-readmission rate is a promising indicator of quality of care, and the correlation between readmissions and other outcomes, such as mortality and LOS, can further enhance its precision. Therefore, the specified indicators coupled with bundling outcome measures can provide a more complete picture of the quality of hospital care. 


\section{How an Intervention Might Work}

In a positive-sum health-care market characterized by positive features (eg, improved value, proper level of competition, suitable market, and correct information about providers), competition generally eliminates inefficiencies that would otherwise yield high production costs, which are ultimately transferred to patients via high health-care prices. $^{4}$ However, competition may not enhance efficiency under a zero sum-based situation in which participants are actively engaged in dividing value instead of creating value. ${ }^{4}$

One theoretical study showed the impact of competition on hospital-quality results of two counteracting forces. ${ }^{28}$ On one hand, competition leads to higher quality, since hospitals can attract high-benefit patients by increasing quality. However, higher quality incurs higher costs, and also attracts more unprofitable patients. If the financial incentive to avoid unprofitable patients is stronger than that to attract high-benefit patients, competition will lead to lower quality. When the price is fixed, competition will lead to higher quality. If hospitals compete on both quality and price, competition will lead to higher quality only when demand is not price-elastic. ${ }^{8}$

Empirical studies have also illustrated that the relationship between competition and hospital quality is ambiguous or even contradictory. ${ }^{1,29-32}$

\section{Why It Is Important to Do This Review}

Basic economics theory supports competition as a mechanism to improve quality of care and contain the cost inflation of medical care for health-care institutions. Numerous studies have been conducted to investigate the impact of increased competition from different perspectives. These have focused on relationships between competition and quality of health care using a range of diverse measures.

Some reviews ${ }^{11,33}$, have tried to summarize the relationship between competition and quality of care. These reviews showed that existing empirical studies have produced inconclusive - sometimes contradictory - findings on the effects of hospital competition (eg, NoH, HHI, demand elasticity, and entrants' market share) and inpatient quality of care (eg, mortality, patient-safety events, and graft failure). These inconsistencies may arise from the use of different methodologies in defining hospital-market competition and quality of care.
To the best of our knowledge, no review has been done using unplanned hospital readmission as an indicator of hospital quality, even though there has been tremendous growth and progress in this field using unplanned readmissions as an indicator in the last two decades. A rigorous systematic review of this kind that takes into account the most recent data is valuable for health-care policymakers. This review is the first in a suite of new reviews formed by splitting the different quality-of-care measures into individual reviews, beginning with readmissions/mortality/LOS at the health care service-provider level.

\section{Methods}

\section{Criteria for Considering Studies for This Review}

We included all published and unpublished studies in any language. Both cross-sectional and longitudinal studies (using balanced/unbalanced panel data) are included. This review did not include qualitative studies. Quantitative studies not using multivariate analysis to control for potential confounders were also excluded. We included all hospitals, regardless of their ownership (public/private) and service type (specialist/comprehensive). We included studies where readmission was one of the outcome variables and competitions were measured only by the HHI. We accepted all authors' definitions of the relevant market for hospital care (hospital market).

The primary outcome of this review was changes in unplanned readmission. Secondary outcomes were changes in mortality rate and changes in LOS. We accepted all author's definition of readmission, if it is considered as unplanned readmission, and we accept all author's definitions of mortality. For cross-sectional studies, all review outcomes are measured by HHI slopes presented in regression models where readmission/mortality rate/LOS or their transformation (eg, logarithmic) was the response and the HHI or its transformation (eg, logarithmic) was one of the predictors.

\section{Search Methods for Identification of Studies}

We searched PubMed, EmBase, Wiley Online Library, Web of Science, Scopus, and JSTOR on September 17, 2019. The search strategies are listed in (Appendix Table A1). Additionally, we reviewed the reference lists in articles that fulfilled our eligibility criteria and relevant reviews. We also searched unpublished potential studies on New 
Economics Papers, which is an announcement service that filters information on new additions to RePEc (Research Papers in Economics) into edited reports. It included not only published papers but also working papers. We completed a PRISMA flowchart to summarize this process.

\section{Data Collection and Analysis}

Titles, abstracts, and full papers were assessed and data extracted independently by two review authors. Disagreements were resolved by discussion, drawing on a third review author where required. We collected outcome data for relevant time points. Where possible, we extracted data on country of origin, sample period, sample size, diseases included, definition of market, market-share indicators, indicator of competition, statistical models, and controlled variables.

Two review authors independently assessed risk of bias for each eligible study using the AXIS appraisal tool for cross-sectional studies, ${ }^{34}$ which addresses five domains (aim, methods, results, discussion, and others) and has been widely used to assess quantitative studies ${ }^{35}$ (see Table A2 in Appendix). Heterogeneity was measured by methodological heterogeneity (such as participants, competition, outcome indicators) and statistical heterogeneity through $\chi^{2}$ tests $(P<0.1$ was considered statistically significant heterogeneity) in conjunction with the $I^{2}$ statistic $(<25 \%$ represented low heterogeneity, $>75 \%$ considerable heterogeneity). ${ }^{36,37}$ Details of included studies were combined in a narrative review according to outcome variables. We considered design and methodological heterogeneity, and pooled slopes extracted from studies that appeared appropriately similar in terms of HHI type (continuous or categorical, transformed or not), empirical model, and outcome type using weighted least squares. ${ }^{38}$

In line with the standard Cochrane methods, we use the GRADE approach to assess the certainty of evidence. ${ }^{39}$ We present the results of the meta-analysis and other studies using a summary of findings (Table 3 ) for all outcomes. Criteria used to grade certainty were:

- For study limitations, we downgraded one level for serious risk of bias if the disease used for measurement of outcome variables had not been used in previous relevant hospital-qualitystudies. As the HHI and outcome variables were calculated by authors based on a secondhand database, it was impossible to blind. We downgraded if the premises of the associating unplanned readmissions with quality were not discussed. We also downgraded one level if endogeneity of the HHI was not controlled.

- For inconsistency of results, we downgraded if there was substantial heterogeneity $\left(I^{2}>50 \%\right)$.

- For indirectness of evidence, we downgraded if there were limited populations or settings in the studies that did not allow us to make generalizations about the findings to other settings relevant to this review.

- For imprecision, we downgraded for small samples and large CIs that included effects in both directions.

\section{Results}

\section{Description of Studies}

The search initially identified 961 records from the six digital databases. A total of 545 articles were included for reviewing title and abstract after duplicates had been removed, of which 17 records were retained for full-text screening. By screening the reference lists of the 17 full texts, four additional records were added. Finally, nine studies were assessed as eligible and the remaining 12 excluded (see Figure 1). As shown in Table 1, five studies were undertaken in the US, ${ }^{40-44}$ two in South Korea, ${ }^{45,46}$ one in Australia, ${ }^{47}$ and one in Taiwan. ${ }^{48}$ Five studies focused on emergency disease, namely heart attack ${ }^{40,41,43}$ and/or stroke ${ }^{42,48}$ two sincluded only minor diseases, one on hemorrhoids, ${ }^{46}$ other on minor nonsurgical heart episodes, ${ }^{47}$ one on all joint-related diseases (eg, knee replacement and hip replacementl), ${ }^{45}$ and one on coronary artery-bypass graft (CABG) surgery, which can be an emergency or not depending on the situation. ${ }^{44}$

In terms of empirical models, four studies had added the HHI to the statistical model as a continuous variable, of which two fit the linear probability model, ${ }^{40,42}$ one applied a multilevel generalized estimate equation model, ${ }^{45}$ and the other used a two-level random-intercept logistic model. ${ }^{47} \mathrm{In}$ the remaining five studies, the HHI was categorized according to either quantiles ${ }^{41,43,44,46}$ or self-definition, ${ }^{48}$ and different models were applied. Of the nine studies, just two ${ }^{41,43}$ used predicted patient flow to define the hospital market, which can avoid the endogeneity problem of the HHI.

As for primary outcomes, four studies reported on the impact of competition on 30-day readmission after discharge, ${ }^{45-48}$ others reported readmission rates within a time longer than 30 days. $^{40-44}$ However, none of them discussed assumptions regarding associations of readmission with quality of care. In terms of secondary outcomes, 


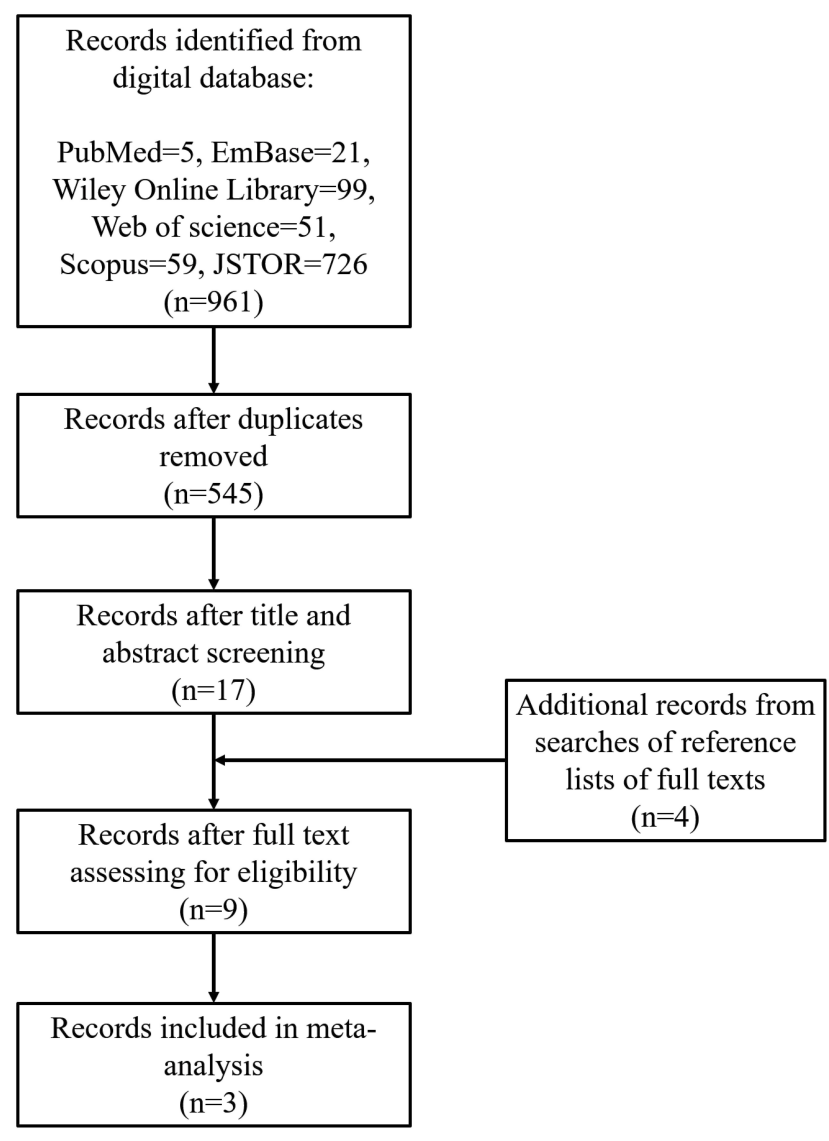

Figure I PRISMA flowchart of screening records.

Notes: PRISMA figure adapted from Liberati A, Altman D, Tetzlaff J, et al. The PRISMA statement for reporting systematic reviews and meta-analyses of studies that evaluate health care interventions: explanation and elaboration. Journal of clinical epidemiology. 2009;62(10). Creative Commons. ${ }^{61}$

seven studies reported on the impact of competition on mortality rate $40-45,47,48$ and two on the influence on LOS. $^{45,46}$ A total of 12 studies were excluded after investigation of the full text. Seven studies had wrong competition and/or quality indicators, ie, their empirical models did not include the HHI as a predictor or readmission as responder. ${ }^{2,28,32,49-52}$ Three recodes were excluded, because the full texts were not available. ${ }^{53-55}$ The other two papers did not study the relationship between competition and hospital quality (Table 2). ${ }^{56,57}$

\section{Risk of Bias in Included Studies}

The quality of the eligible studies in this review appeared to range from moderate to strong, as all nine studies met most of the AXIS criteria, ${ }^{34}$ ie, $14-20$ of 20 (mean 17.6 \pm 1.9 , Appendix Table A3). Of the nine studies included, two met 20 of 20 items. $^{41,42}$ Of those that missed criteria, most did not take measures to address, categorize, or describe the deleted data $(n=5,55.6 \%)$. Three studies ${ }^{46-48}$ did not measure the
HHI or outcome variables correctly using instruments/measurements published previously. Kim et $\mathrm{al}^{46}$ and Palangkaraya et $\mathrm{al}^{47}$ measured outcomes based on hemorrhoids and minor nonsurgical heart disease, respectively, which had not been used in relevant hospital-qualitystudies previously. Liao et $\mathrm{al}^{48}$ classified a market into three types (high, moderate, and low competition) according to two HHI cutoff points $(1,000$ and 1,800) without valid reference.

\section{Effects of Competition \\ Primary Outcome: Changes in Unplanned Readmission}

All nine studies reported this primary outcome, while only three were included in meta-analysis: ${ }^{42,45,47} \mathrm{Kim}$ et $\mathrm{al}^{45}$ focused on 30-day readmission of patients with jointrelated diseases in South Korea, Palangkaraya et $\mathrm{al}^{47}$ looked at 30-day readmission of patients with minor and nonsurgical heart disease in Australia, and Ho and Hamilton $^{42}$ studied 1-year readmission rates for heart attack and stroke patients in the US. Only these three studies used the HHI as a continuous variable, and the partial effect of the HHI (its initial form, rather than the transformed $\mathrm{HHI}$ ) were reported. The statistics implied no significant heterogeneity in effect sizes among these three studies $\left(I^{2}=0\right)$. The pooled result showed that it was uncertain whether or not hospital competition reduced readmission rates $(\beta=0.02, P=0.06$; Figure 2$)$. We considered these data to be very low-certainty evidence, as they were all observational studies with high heterogeneity (Table 3).

Rather than using the HHI as a continuous variable, five of the remaining six studies categorized the HHI as discrete data when fitting regression models. ${ }^{41,43,44,46,48}$ Chou et al, ${ }^{44}$ Kessler et al, ${ }^{43}$ and Kim et $\mathrm{al}^{46}$ categorized market competition as high, moderate, or low using quantiles of the HHI, Liao et $\mathrm{al}^{48}$ categorized market competition as high atHHI $<1,000$, less competitive at $\mathrm{HHI}$ $>1,800$, and otherwise moderately competitive, while Kessler et al (2000) ${ }^{41}$ categorized competition into five categories according to quantiles of the HHI. The remaining one ${ }^{40}$ converted the HHI to its natural $\log$ form, although it was a continuous variable. Consequently, it was impossible to pool the results of these six studies, and they are reported narratively. Meanwhile, these studies showed various effects between competition and readmission.

Among the six studies, three reported that hospital competition had no effect on readmission. Chou et $\mathrm{al}^{44}$ focused on 76,862 patients who underwentCABG 
Table I Characteristics of Included Studies

\begin{tabular}{|c|c|c|c|}
\hline & Sample information & Methods & Outcomes \\
\hline Chou et al ${ }^{44}$ & $\begin{array}{l}\text { Setting: Pennsylvania, USA } \\
\text { Data source: inpatient database of } \\
\text { Pennsylvania Health Care Cost Containment } \\
\text { Council (PHC4) } \\
\text { Sample period: } 1995-2004 \\
\text { Sample size: } 76,862 \\
\text { Treatment: Coronary artery-bypass graft } \\
\text { (CABG) surgery }\end{array}$ & $\begin{array}{l}\text { Market definition: Variable radius methods } \\
\text { (determined by the area from which the } \\
\text { hospital draws } 75 \% \text { of its patients) } \\
\text { Market share indicator: predicted volume of } \\
\text { patients } \\
\text { Competition indicator: HHI (categorized } \\
\text { market competition as most competitive, } \\
\text { competitive or low competitive using quantiles } \\
\text { of HHI) } \\
\text { Statistical model: Linear probability model } \\
\text { Included confounders } \\
\text { I) hospital level: no } \\
\text { 2) physician level: no } \\
\text { 3) individual level: Age, sex, Ethnicity, } \\
\text { Admitted through emergency sector, Medicare } \\
\text { HMO enrolee or not, Patient admission source, } \\
\text { Distance to the closest CABG hospital, } \\
\text { Charlson index } \\
\text { 4) others: Predicted number of hospital beds, } \\
\text { Number of teaching hospitals (for each zip } \\
\text { code), Year, Zip code, Admitting hospital }\end{array}$ & $\begin{array}{l}\text { Primary outcome } \\
\text { Readmission (I year): not reported the main } \\
\text { effect of competition, only reported the } \\
\text { interaction between categorized competition } \\
\text { indicators and other variables, all the estimators } \\
\text { of these interactions were not significant at } \\
\alpha=0.05 \\
\text { Secondary outcome } \\
\text { Mortality (in hospital): not reported the } \\
\text { main effect of competition, only reported the } \\
\text { interaction between categorized competition } \\
\text { indicators and other variables, authors said the } \\
\text { mortality rate for the more severely ill in the } \\
\text { most competitive markets was approximately } \\
\text { ( } 0.0072+0.0003) \text { points lower, while for } \\
\text { severely ill patients in competitive markets the } \\
\text { reduction was }(0.0095+0.0032 \text { ) points } \\
\text { Length of hospital stay: not reported }\end{array}$ \\
\hline Dunn et $\mathrm{a}^{40}$ & $\begin{array}{l}\text { Setting: USA } \\
\text { Data source: MarketScan Commercial Claims } \\
\text { and Encounters database, SK\&A physician } \\
\text { database } \\
\text { Sample period: } 2005-2008 \\
\text { Sample size: } 12,020 \\
\text { Disease: first-time heart attack (ICD9-CM } \\
410 \text { ) treated in the emergency room, } \\
\text { commercially insured patients aged } 19-63 \text { years }\end{array}$ & $\begin{array}{l}\text { Market definition: Fixed-travel-time ( } 80 \\
\text { minutes driving time) } \\
\text { Market share indicator: volume of patients } \\
\text { Competition indicator: log(HHI) } \\
\text { Statistical model: Linear probability model } \\
\text { Included confounders } \\
\text { I) hospital level: no } \\
\text { 2) physician level: no } \\
\text { 3) individual level: Age, Interaction of sex and } \\
\text { age dummies, Patient's type of insurance carrier, } \\
\text { Whether the patient was ever admitted to an } \\
\text { inpatient hospital before the heart attack } \\
\text { occurred, Charlson comorbidity index, Specific } \\
\text { type of heart attack } \\
\text { 4) others: Whether the data source was from } \\
\text { an employer, Number of cardiologists per } \\
\text { capita, Number of cardiologists per firm, } \\
\text { Number of hospitals per capita, Number of } \\
\text { university hospitals per capita, Population } \\
\text { density, Hospital costs per employee, Median } \\
\text { house value, Median house rental, Median } \\
\text { income of the patient's county of residence, } \\
\text { Prediction of the fraction of heavy smokers, the } \\
\text { Fraction of obese, Number of non- } \\
\text { cardiovascular-related treated conditions per } \\
\text { capita, Amount of non-cardiovascular-related } \\
\text { services per episode of care, Concentration of } \\
\text { the insurance market. }\end{array}$ & $\begin{array}{l}\text { Primary outcome } \\
\text { Readmission ( } 90-365 \text { day): }-0.002(0.001) \\
\text { Secondary outcome Mortality }(90 \text { days } \\
\text { after admission): }-0.002(0.002) \\
\text { Length of hospital stay: not reported }\end{array}$ \\
\hline
\end{tabular}

(Continued) 
Table I (Continued).

\begin{tabular}{|c|c|c|c|}
\hline & Sample information & Methods & Outcomes \\
\hline $\begin{array}{l}\text { Kessler and } \\
\text { McClellan }\end{array}$ & $\begin{array}{l}\text { Setting: USA } \\
\text { Data source: longitudinal data on cohorts of } \\
\text { elderly Medicare beneficiaries, American } \\
\text { Hospital Association (for hospital information) } \\
\text { Sample period: 1985, 1988, 1991, and 1994 } \\
\text { Sample size: } 146,569 \text { in 1985, 137,879 in } \\
\text { 1988, } 145,555 \text { in 1991, } 143,308 \text { in 1994 } \\
\text { Disease: heart attacks }\end{array}$ & $\begin{array}{l}\text { Market definition: Predicted patient flow } \\
\text { Market share indicator: volume of patients } \\
\text { Competition indicator: HHI (categorized as } \\
\text { very low, low, high and very high according to } \\
\text { quantiles of HHI) } \\
\text { Statistical model: Linear probability model } \\
\text { Included confounders } \\
\text { I) hospital level: Densities of hospitals of } \\
\text { different sizes, Ownership statuses, Teaching } \\
\text { statuses, The travel distance to the hospital } \\
\text { nearest to zip code } \\
\text { 2) physician level: no } \\
\text { 3) individual level: age,sex, black/nonblack, } \\
\text { interactions between year and each of the age, } \\
\text { sex, race indicators. } \\
\text { 4) others: the size of individual's MSA }\end{array}$ & $\begin{array}{l}\text { Primary outcome } \\
\text { Readmission ( } \mathrm{I} \text { year): Very high } \mathrm{HHI}: 0.54 \\
(0.33) \text {, high HHI: } 0.23(0.28) \text {, low } \mathrm{HHI}: 0.16 \\
(0.2 \mathrm{I}) \text {, Ref: very low HHI. } \\
\text { Secondary outcome } \\
\text { Mortality (I year): Very high } \mathrm{HHI}: 1.46^{*} \\
(0.69) \text {, high HHI: } 0.46(0.57) \text {, low } \mathrm{HHI}: 0.65 \\
(0.44) \text {, Ref: very low HHI. } \\
\text { Length of hospital stay: not reported } \\
\text { Notes: Two statistical models were fit basing } \\
\text { on sample from pre- and post- } 1990 \\
\text { respectively, we only included the coefficient of } \\
\text { last time point. }\end{array}$ \\
\hline $\begin{array}{l}\text { Kessler and } \\
\text { Geppert }^{43}\end{array}$ & $\begin{array}{l}\text { Setting: USA } \\
\text { Data source: individual-level longitudinal } \\
\text { Medicare claims data from the Centers for } \\
\text { Medicare } \\
\text { and Medicaid Services (CMS), CMS‘s HISKEW } \\
\text { enrolment files for demographic characteristics, } \\
\text { US hospital characteristics collected by the } \\
\text { American Hospital Association, hospital-system } \\
\text { database } \\
\text { Sample period: 1985-1996 } \\
\text { Sample size: not clear, but 158,067 patients in } \\
\text { 1985 and I55,707 in 1996 } \\
\text { Disease: heart attacks }\end{array}$ & $\begin{array}{l}\text { Market definition: Predicted patient flow } \\
\text { Market share indicator: volume of patients } \\
\text { Competition indicator: HHI (categorized as } \\
\text { very low, low, high and very high according to } \\
\text { quantiles of HHI) } \\
\text { Statistical model: Linear probability model } \\
\text { Included confounders } \\
\text { I) hospital level: Densities of hospitals of } \\
\text { different sizes, Ownership statuses, Teaching } \\
\text { statuses, The travel distance to the hospital } \\
\text { nearest to zip code } \\
\text { 2) physician level: no } \\
\text { 3) individual level: Age, sex, Black/nonblack } \\
\text { race, Interactions between year and each of the } \\
\text { age, sex, race indicators, Interactions between } \\
\text { year and each of the age, sex, and race } \\
\text { indicators, patient has health status } \\
\text { 4) others: no }\end{array}$ & $\begin{array}{l}\text { Primary outcome } \\
\text { Readmission (I year): } \\
\text { for Low-risk Patients } \\
\text { Very high HHI: }-0.014(0.132) \text {, Middle high HHI: } \\
-0.10 \text { I ( } 0.096) \text {, Ref: Low HHI. } \\
\text { for High-risk Patients } \\
\text { Very high HHI: }-0.135 \text { ( } 0.156) \text {, Middle high HHI: } \\
-0.233 \text { ( } 0.1 \text { I } 2) \text {, Ref: Low HHI. } \\
\text { Secondary outcome } \\
\text { Mortality (I year): } \\
\text { for Low-risk Patients } \\
\text { Very high HHI: } 0.22 \mathrm{I} \text { ( } 0.28 \mathrm{I}) \text {, Middle high HHI: } \\
-0.083 \text { ( } 0.197) \text {, Ref: Low HHI. } \\
\text { for High-risk Patients } \\
\text { Very high HHI: } 0.822 \text { ( } 0.319) \text {, Middle high HHI: } \\
0.496 \text { ( } 0.230) \text {, Ref: Low HHI. } \\
\text { Length of hospital stay: not reported }\end{array}$ \\
\hline Kim et $\mathrm{al}^{45}$ & $\begin{array}{l}\text { Setting: South Korea } \\
\text { Data source: records of inpatient health- } \\
\text { insurance claims } \\
\text { Sample period: from November I, } 201 \mathrm{I} \text { to } \\
\text { May } 3 \mathrm{I}, 2012 \\
\text { Sample size: } 279,847 \text { patients from } 85 \mathrm{I} \\
\text { hospitals } \\
\text { Disease: All joint-related diseases included } \\
\text { both surgical and nonsurgical procedures } \\
\text { (surgical procedures included shoulder surgery, } \\
\text { semilunar cartilage surgery, knee replacement } \\
\text { arthroplasty, hip replacement arthroplasty, and } \\
\text { all other joint surgeries) }\end{array}$ & $\begin{array}{l}\text { Market definition: Administrative districts } \\
\text { Market share indicator: total charges for } \\
\text { patients with joint disease } \\
\text { Competition indicator: HHI by } 100 \text { points } \\
\text { Statistical model: Multilevel generalized } \\
\text { estimating equation models } \\
\text { Included confounders } \\
\text { I) hospital level: Hospital type (small, } \\
\text { research, general), HHI × Hospital type, } \\
\text { Hospital ownership, Beds, Specialist, Nurses, } \\
\text { Teaching hospital, Beds occupancy rate } \\
\text { 2) physician level: no } \\
\text { 3) individual level: Age, sex, Clinical } \\
\text { complexity level, Surgical procedures } \\
\text { 4) others: Per capita GRDP }\end{array}$ & $\begin{array}{l}\text { Primary outcome } \\
\text { Readmission ( } 30 \text { days): } 0.00012 \text { (not } \\
\text { reported SE, the P value was } 0.835 \text { ), SE was } \\
\text { calculated by the authors as } 0.057 \text { I basing on } \\
\text { the announced estimator and P value. } \\
\text { Secondary outcome } \\
\text { Mortality ( } 30 \text { days after admission): - } \\
0.00004 \text { (not reported SE, the P value was } \\
0.111 \text { ) SE was calculated by the authors as } \\
0.0025 \text { basing on the announced estimator and } \\
P \text { value. } \\
\text { Length of hospital stay (log } \\
\text { transformation): } 0.023 \text { (not reported SE, the } \\
P \text { value was reported as }<0.000 \mathrm{I} \text { ) }\end{array}$ \\
\hline
\end{tabular}

(Continued) 
Table I (Continued).

\begin{tabular}{|c|c|c|c|}
\hline & Sample information & Methods & Outcomes \\
\hline Kim et $\mathrm{al}^{46}$ & $\begin{array}{l}\text { Setting: South Korea } \\
\text { Data source: Inpatient health insurance claims } \\
\text { Sample period: from July } 2011 \text { to July } 2014 \\
\text { Sample size: } 821,912 \text { hospitalizations and } \\
\text { I,742 hospitals } \\
\text { Disease: hemorrhoids (South Korean DRG } \\
\text { codes: G1020, G1040, G1050 and G1060) }\end{array}$ & $\begin{array}{l}\text { Market definition: Administrative districts } \\
\text { Market share indicator: total discharged } \\
\text { patients for hemorrhoid } \\
\text { Competition indicator: HHI (categorized } \\
\text { market competition as high, moderate or low } \\
\text { using quantiles of HHI) } \\
\text { Statistical model: Generalized estimating } \\
\text { equation models } \\
\text { Included confounders } \\
\text { I) hospital level: Hospital type (Clinic, } \\
\text { Hospital, General hospital, Tertiary hospital), } \\
\text { Case mix index, Number of I00 beds, Number } \\
\text { of doctor per I00 beds, Number of nurse per } \\
\text { l00 beds, Number of pharmacist, Ownership } \\
\text { status (Private, Public), Introduction of DRG, } \\
\text { Teaching status, hospital location (Urban, } \\
\text { Rural), Status } \\
\text { 2) physician level: no } \\
\text { 3) individual level: Age, sex, Clinical } \\
\text { complexity level, LOS } \\
\text { 4) others: Year }\end{array}$ & $\begin{array}{l}\text { Primary outcome } \\
\text { Readmission ( } 30 \text { days): } \\
\text { high competition OR } 0.95 \text { (not reported SE, the } \\
\text { P value was } 0.0277 \text { ), } \\
\text { low competition OR I.2I (not reported SE, the } \\
\text { P value was reported as }<0.000 I \text { ), Ref: } \\
\text { moderate competition. } \\
\text { Secondary outcome } \\
\text { Mortality: not report } \\
\text { Length of hospital stay (log } \\
\text { transformation): } \\
\text { high competition } 0.0100 \text { (not reported SE, the P } \\
\text { value was reported as }<0.000 \mathrm{I} \text { ), } \\
\text { low competition } 0.0002 \text { (not reported SE, the P } \\
\text { value was } 0.945 \mathrm{I} \text { ), } \\
\text { Ref: moderate competition. }\end{array}$ \\
\hline Liao et $\mathrm{al}^{48}$ & $\begin{array}{l}\text { Setting: Taiwan } \\
\text { Data source: NHI Research Database } \\
\text { (NHIRD), and the National Hospital and } \\
\text { Services Survey (NHSS) } \\
\text { Sample period: from } 01 / 01 / 1997 \text { to } 31 / 12 / \\
2007 \\
\text { Sample size: } 327,120 \text { ( } 247,379 \text { ischemic and } \\
79,74 \text { I hemorrhagic stroke) patients } \\
\text { Disease: Stroke patients (hemorrhagic stroke: } \\
\text { ICD-9-CM codes } 430 \text { and } 431 \text {, ischemic stroke: } \\
\text { ICD-9-CM codes } 433 \text { and } 434 \text { ) }\end{array}$ & $\begin{array}{l}\text { Market definition: } 16 \text { medical care networks } \\
\text { that defined by Department of Health were } \\
\text { regarded as the designated health care market } \\
\text { area } \\
\text { Market share indicator: the percentage of } \\
\text { inpatient days attributable to stroke patients of } \\
\text { a hospital in the defined market } \\
\text { Competition indicator: HHI (categorized } \\
\text { market competition as highly (HHI<1000), } \\
\text { moderately competitive (I000 HHI I800) and } \\
\text { less competitive (highly concentrated, } \\
\text { HHI>I800).) } \\
\text { Statistical model: Pooled time-series cross- } \\
\text { sectional analysis with a fixed-effects model } \\
\text { Included confounders } \\
\text { I) hospital level: Ownership (Private, Public, } \\
\text { Non-for-profit proprietary), Accreditation level } \\
\text { (Medical centre, Regional hospital, District } \\
\text { hospital, Teaching hospital) } \\
\text { 2) physician level: Years of practice as a } \\
\text { specialist, Service volume, Proportion of males } \\
\text { 3) individual level: Age, sex, Disease severity } \\
\text { 4) others: no }\end{array}$ & $\begin{array}{l}\text { Primary outcome } \\
\text { Readmission ( } 30 \text { days): } \\
\text { hemorrhagic stroke } \\
\text { Moderately competitive } 0.0117 \text { ( } 0.0093) \text {, } \\
\text { Less competitive } 0.0092 \text { ( } 0.0075) \text {, Ref: Highly } \\
\text { competitive. } \\
\text { Ischemic stroke } \\
\text { Moderately competitive } 0.0045 \text { ( } 0.0053) \text {, } \\
\text { Less competitive } 0.0043 \text { ( } 0.0042) \text {, Ref: Highly } \\
\text { competitive. } \\
\text { Secondary outcome } \\
\text { Mortality (in hospital): } \\
\text { hemorrhagic stroke } \\
\text { Moderately competitive }-0.0702 \text { ( } 0.0135) \text {, } \\
\text { Less competitive }-0.063 \text { I }(0.0107) \text {, Ref: Highly } \\
\text { competitive. } \\
\text { ischemic stroke } \\
\text { Moderately competitive }-0.0070 \text { (0.0023), } \\
\text { Less competitive }-0.0040 \text { ( } 0.0019) \text {, Ref: Highly } \\
\text { competitive. } \\
\text { Length of hospital stay: } \\
\text { not reported }\end{array}$ \\
\hline
\end{tabular}

(Continued) 
Table I (Continued).

\begin{tabular}{|c|c|c|c|}
\hline & Sample information & Methods & Outcomes \\
\hline $\begin{array}{l}\text { Palangkaraya } \\
\text { et al }{ }^{47}\end{array}$ & $\begin{array}{l}\text { Setting: State of Victoria, Australia } \\
\text { Data source: hospital administration data } \\
\text { Sample period: from 2000/200I to 2004/ } \\
2005 \\
\text { Sample size: I57,427 admission episodes } \\
\text { Disease: minor, nonsurgical, heart episodes } \\
\text { according to seven DRG codes: } \\
\text { F65 (peripheral vascular disorders), F66 } \\
\text { (coronary atherosclerosis), F67 (hypertension), } \\
\text { F69 (valvular disorder), F7I (non-major } \\
\text { arrhythmia and conduction disorders), F72 } \\
\text { (unstable angina), F73 (syncope and collapse). }\end{array}$ & $\begin{array}{l}\text { Market definition: Actual patient flow } \\
\text { Market share indicator: volume of inpatient } \\
\text { Competition indicator: (I-HHI) } \\
\text { Statistical model: two-level random- } \\
\text { intercept logistic model } \\
\text { Included confounders } \\
\text { I) hospital level: Hospital overall caseload } \\
\text { volume, Proportion of admissions with no } \\
\text { comorbidity, Proportion of admissions with no } \\
\text { ICU stay, Proportion of admissions with private } \\
\text { insurance, teaching hospital } \\
\text { 2) physician level: no } \\
\text { 3) individual level: Age (years), Male, } \\
\text { Australian born, with private hospital insurance, } \\
\text { Charlson comorbidity index, first-time heart } \\
\text { diagnosis, admitted via emergency department, } \\
\text { same-day separation, transfers between } \\
\text { hospitals, with complexities and complications } \\
\text { 4) others: number of competitors }\end{array}$ & $\begin{array}{l}\text { Primary outcome } \\
\text { Readmission ( } 30 \text { days): } \\
-0.3238(0.1216) \text { (average partial effect of (I- } \\
H H I)-0.018(0.009 I)) \\
\text { Secondary outcome } \\
\text { Mortality ( } 30 \text { days): } \\
0.2625(0.2017) \text { (average partial effect of }(\mathrm{I}- \\
H H I) 0.005(0.0038)) \\
\text { Length of hospital stay: } \\
\text { not reported }\end{array}$ \\
\hline Ho et $\mathrm{al}^{42}$ & $\begin{array}{l}\text { Setting: California, USA } \\
\text { Data source: American Hospital Association } \\
\text { AHA Annual Survey of Hospitals, California } \\
\text { Office of State wide Health Planning and } \\
\text { Development OSHPD discharge data } \\
\text { Sample period: from } 1992 \text { to } 1995 \\
\text { Sample size: } 256,193 \text { heart attack patients (in } \\
46 \text { I hospitals), } 268,506 \text { stroke patients (in } 476 \\
\text { hospitals) } \\
\text { Disease: heart attack and stroke }\end{array}$ & $\begin{array}{l}\text { Market definition: Health Service Areas } \\
\text { formed basing on travel patterns between } \\
\text { counties by Medicare beneficiaries for routine } \\
\text { hospital care (referred to definition from other } \\
\text { study) } \\
\text { Market share indicator: not reported } \\
\text { Competition indicator: HHI } \\
\text { Statistical model: linear probability model } \\
\text { Included confounders } \\
\text { I) hospital level: Merger, Independent } \\
\text { acquired, System acquired, Patient volume, } \\
\text { Merger*HHI, Independent acquired*HHI, } \\
\text { System acquired*HHI } \\
\text { 2) physician level: no } \\
\text { 3) individual level: Ages (categorical } \\
\text { variable), sex, Races (black, Hispanic, Asian and } \\
\text { other races), Comorbidities, Length of stay, } \\
\text { Transfers, Medi-Cal, Private insurance, Self-pay, } \\
\text { Indigent, Other payment } \\
\text { 4) others: no }\end{array}$ & $\begin{array}{l}\text { Primary outcome } \\
\text { Readmission ( } 90 \text { days): } \\
-0.059 \text { (not reported SE, the t-statistics was } \\
-0.74 I \text { ) } \\
\text { Note: SE was calculated by the authors as } \\
0.0796 \text { basing on the announced estimator and } \\
\text { t-statistics. } \\
\text { Secondary outcome } \\
\text { Mortality ( } 30 \text { days): } \\
\text { not reported } \\
\text { Length of hospital stay: } \\
\text { not reported }\end{array}$ \\
\hline
\end{tabular}

surgery in the US, and showed that the interaction effect between the HHI and other variables was not significant at $\alpha=0.05$. Liao et $\mathrm{al}^{48}$ used data of 327,120 stroke (247,379 ischemic and 79,741 hemorrhagic) patients from Taiwan, and found no significant difference among hospitals in moderately (hemorrhagic stroke, $\beta=0.0117$, SE 0.0093; ischemic stroke, $\beta=0.0045$, SE 0.0053) and less competitive markets (hemorrhagic stroke, $\beta=0.0092$, SE 0.0075; ischemic stroke, $\beta=0.0043$, SE 0.0042) in terms of 30-day readmission rate when compared with hospitals located in highly competitive markets.
Kessler et $\mathrm{al}^{41}$ investigated 287,863 patients who went to hospitals for acute myocardial infarction (AMI) in 1991 and 1994 in the US, and did not find a significant difference in 1-year readmission rate in markets with very high $(\beta=0.54$, SE 0.33$)$, high $(\beta=0.23$, SE 0.28$)$ and low HHI $(\beta=0.16$, SE 0.21) compared with markets with very low HHI.

Kim et $\mathrm{al}^{46}$ used national data from July 2011 to July 2014 for patients admitted to hospitals due to hemorrhoids in South Korea. They reported a lower risk of readmission in high-competition areas (OR 0.95, 
Table 2 Characteristics of Excluded Studies

\begin{tabular}{|c|c|}
\hline & Reasons for Exclusion \\
\hline Aggarwal et al ${ }^{49}$ & Competition indicator not $\mathrm{HHI}$ \\
\hline Berta et $\mathrm{al}^{32}$ & Included multiple quality indicators, unable to obtain readmission data separately \\
\hline Brekke et $\mathrm{al}^{28}$ & Competition indicator not $\mathrm{HHI}$, the quality indicators did not include readmission \\
\hline Chhatre et $\mathrm{al}^{53}$ & Meeting abstract only, full text not available \\
\hline Chua et $\mathrm{a}^{56}$ & Not about relationship between competition and quality \\
\hline Hayford et $\mathrm{al}^{50}$ & Health-outcome indicators did not include readmission \\
\hline Joynt et $\mathrm{al}^{54}$ & Meeting abstract only, full text not available \\
\hline Lee et $\mathrm{al}^{55}$ & Full text not available \\
\hline Leleu et $\mathrm{al}^{57}$ & Not a hospital quality-related study \\
\hline Longo et $\mathrm{al}^{2}$ & Outcomes did not include readmission \\
\hline Mutter et $\mathrm{al}^{51}$ & Quality indicators did not include readmission \\
\hline Siciliani et $\mathrm{al}^{52}$ & Regression of readmission to $\mathrm{HHI}$ not established \\
\hline
\end{tabular}

$P=0.0277)$, and higher risk in low-competition areas (OR 1.21, $P<0.0001)$ compared with moderate-competition areas. That is a negative relationship between competition and 30-day readmission. Another study suggested that competition was positively correlated with readmissions:40 Dunn et $\mathrm{al}^{40}$ (12,020 participants) found that an increase of one unit in $\log _{\mathrm{HHI}}$ would cause a decrease of 0.002 (SE 0.001) units in readmission rate during 90-365 days after discharge for AMI patients. The remaining study ${ }^{43}$ reported subgroup data, and showed competition had no relationship with readmission for patients with lower-risk AMI (very high vs low HHI, $\beta=-0.014$, SE 0.132; high vs low HHI, $\beta=-0.101$, SE 0.096), while there was a positive relationship for higher-risk AMI patients (very high vs low HHI, $\beta=-0.135$, SE 0.156 ; high vs low HHI, $\beta=-0.233$, SE 0.112) in the US.

\section{Secondary Outcome: Changes in Mortality}

Seven studies reported on the impact of competition on the risk of mortality. ${ }^{40,41,43-45,47,48} \mathrm{Kim}$ et $\mathrm{al}^{45}$ and Palangkaraya et $\mathrm{al}^{47}$ reported 30-day mortality for jointrelated diseases and minor and nonsurgical heart disease, respectively. These two studies used the HHI as a continuous variable, and reported partial effects with the index. Upon pooling, here was high heterogeneity between the two studies $\left(I^{2}=75 \%, P<0.05\left[\chi^{2}\right.\right.$ test $\left.]\right)$. Therefore, the random-effect model was used for data synthesis, and it was uncertain whether competition had an effect on mortality $(\beta=-0, P=0.99$; Figure 3 ). We considered these data to be very low-certainty evidence, due to high risk of bias, inconsistency and indirectness.

The remaining five studies were unable to be included in meta-analysis for the same reasons we mentioned, ie,

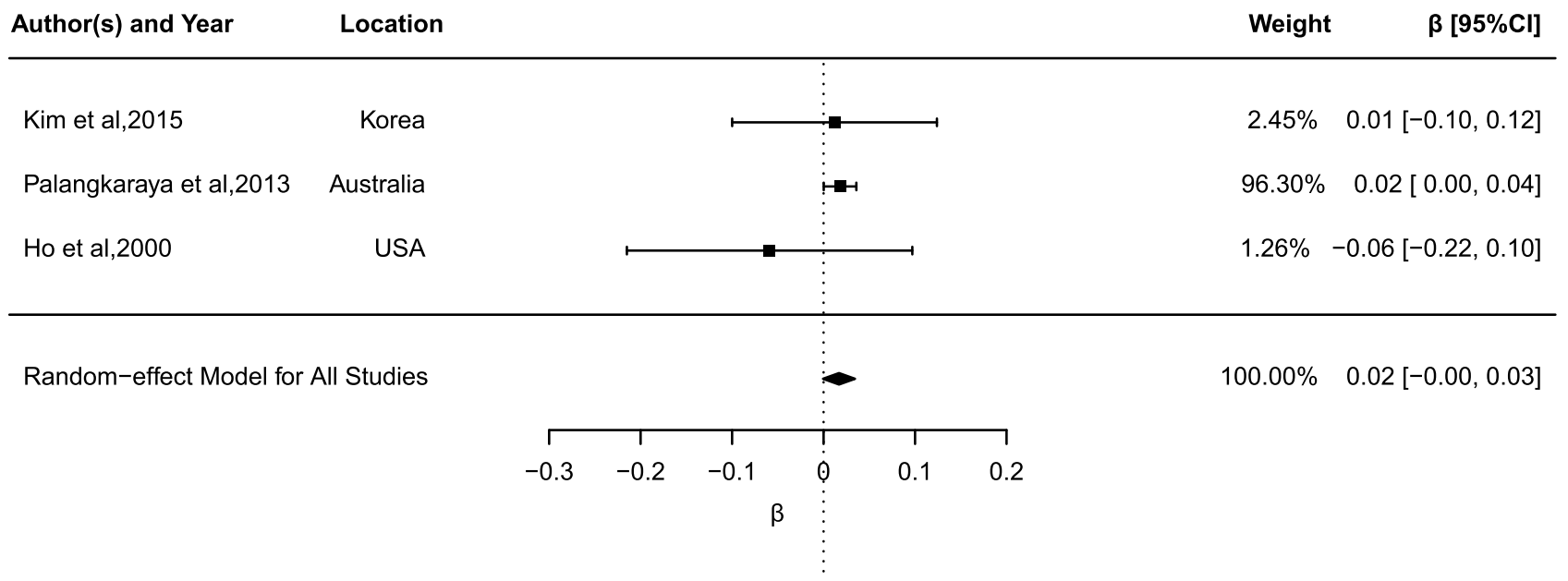

Figure 2 Forest plots of the effect of hospital competition on readmission.

Notes: The weight given to each study was the inverse of the SE of the effect estimate (inverse-variance method); $\beta>0$ implies higher HHI (lower degree of competition) is associated with higher readmission rate. 
Table 3 Summary of Findings

\begin{tabular}{|c|c|c|c|c|}
\hline & Impact & $\begin{array}{l}\text { Participants } \\
\text { (studies), n }\end{array}$ & $\begin{array}{l}\text { Certainty of } \\
\text { evidence } \\
\text { (GRADE)* }\end{array}$ & Comments \\
\hline \multicolumn{5}{|l|}{ Meta-analysis } \\
\hline $\begin{array}{l}\text { Changes } \\
\text { in readmission rate }\end{array}$ & $\begin{array}{l}\beta(95 \% \mathrm{Cl}) \text { of } \mathrm{HHI}: \\
0.02(-0.00 \text { to } 0.03)\end{array}$ & $\begin{array}{l}532,125 \\
\text { (three) }\end{array}$ & $\begin{array}{l}\oplus \oplus \Theta \Theta^{\mathrm{a}, \mathrm{b}} \\
\text { Very low }\end{array}$ & $\begin{array}{l}\text { Uncertain whether or not } \\
\text { hospital competition reduces } \\
\text { readmission rate }\end{array}$ \\
\hline $\begin{array}{l}\text { Changes in } \\
\text { mortality rate }\end{array}$ & $\begin{array}{l}\beta(95 \% \mathrm{Cl}) \text { of } \mathrm{HHI}: \\
-0.00(-0.0 \mathrm{I} \text { to } 0.0 \mathrm{I})\end{array}$ & $\begin{array}{l}437,274 \\
\text { (two) }\end{array}$ & $\begin{array}{l}\oplus \Theta \Theta \Theta^{\mathrm{a}-\mathrm{c}} \\
\text { Very low }\end{array}$ & $\begin{array}{l}\text { Uncertain whether or not } \\
\text { hospital competition reduces } \\
\text { mortality rate }\end{array}$ \\
\hline Changes in LOS & - & - & - & - \\
\hline \multicolumn{5}{|l|}{ Others } \\
\hline $\begin{array}{l}\text { Changes in } \\
\text { readmission rate }\end{array}$ & 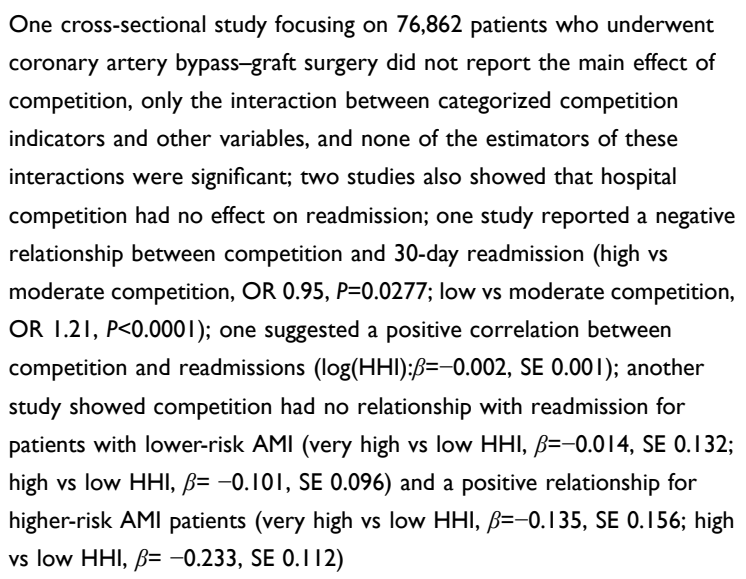 & $\begin{array}{l}\text { I,709,55। } \\
\text { patients } \\
(\text { six })\end{array}$ & $\begin{array}{l}\oplus \Theta \Theta \Theta^{\mathrm{a}, \mathrm{d}} \\
\text { Very low }\end{array}$ & $\begin{array}{l}\text { Uncertain whether or not } \\
\text { hospital competition reduces } \\
\text { readmission rate }\end{array}$ \\
\hline $\begin{array}{l}\text { Changes in } \\
\text { mortality rate }\end{array}$ & $\begin{array}{l}\text { Three studies focused on patients with } \mathrm{AMI} \text { in the US, of which one } \\
\text { showed competition was not associated with mortality, one showed I- } \\
\text { year mortality was } \mathrm{I} .46 \text { points higher in markets with very high } \mathrm{HHI} \text { than } \\
\text { markets with very low } \mathrm{HHI} \text {, with no difference in markets with high or } \\
\text { low } \mathrm{HHI} \text { compared to markets with very low } \mathrm{HHI} \text {; the remaining one } \\
\text { found that competition was not correlated with mortality rate for low- } \\
\text { risk AMI patients, while it was negatively correlated with mortality for } \\
\text { high-risk AMI patients; another two studies investigated the impact of } \\
\text { competition on in-hospital mortality, and one showed higher competition } \\
\text { reduced mortality rate, while the other showed the opposite impact }\end{array}$ & $\begin{array}{l}1,018,639 \\
\text { (five) }\end{array}$ & $\begin{array}{l}\oplus \ominus \Theta \Theta^{\mathrm{a}, \mathrm{e}} \\
\text { Very low }\end{array}$ & $\begin{array}{l}\text { Uncertain whether or not } \\
\text { hospital competition reduces } \\
\text { mortality rate }\end{array}$ \\
\hline Changes in LOS & $\begin{array}{l}\text { Two studies conducted in South Korea: one showed competition } \\
\text { shortened LOS, while the other showed LOS was higher in high- } \\
\text { competition areas compared with moderate-competition areas, but no } \\
\text { difference between low- and moderate-competition areas in terms of LOS }\end{array}$ & $\begin{array}{l}\text { I,101,759 } \\
\text { patients (two) }\end{array}$ & $\begin{array}{l}\oplus \ominus \ominus \Theta^{\text {a,f }} \\
\text { Very low }\end{array}$ & $\begin{array}{l}\text { Uncertain whether or not } \\
\text { hospital competition shortens } \\
\text { LOS }\end{array}$ \\
\hline
\end{tabular}

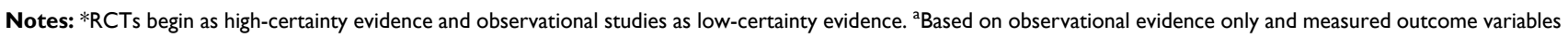
based on diseases that have not been used in previous relevant hospital-quality studies. The premises of associating unplanned readmissions with quality were not discussed, and the endogeneity of $\mathrm{HHI}$ was not controlled. We have not downgraded further due to the low GRADE already reflecting the challenges in inferring causality from observational data. ${ }^{b}$ Downgraded one level for inconsistency: highly heterogeneous $\left(l^{2}=75 \%\right)$ or very heterogeneous studies were included). ${ }^{c}$ Downgraded one level for indirectness. Only two studies: one in South Korea and the other in Victoria, Australia. It was not possible to make broad generalizations to other settings. ${ }^{\mathrm{d}}$ Downgraded one level for indirectness. Four of the six studies conducted in the US. It was not possible to make broad generalizations to other settings. ${ }^{e}$ Downgraded one level for indirectness. All studies conducted in the US, except one conducted in Taiwan. It was not possible to make broad generalizations to other settings. ${ }^{f}$ Downgraded one level for indirectness. Only two studies, both conducted in South Korea. It was not possible to make broad generalizations to other settings.

rather than providing continuous data, they converted the form of the HHI (such as logarithm transformation or discretization). Therefore, we narratively summarised their results. Dunn et $a,^{40}$ focusing on AMI patients in the US, did not find a statistically significant relationship between competition and mortality $\left(\log _{\mathrm{HHI}}: \beta=0.002, \mathrm{SE}\right.$ 


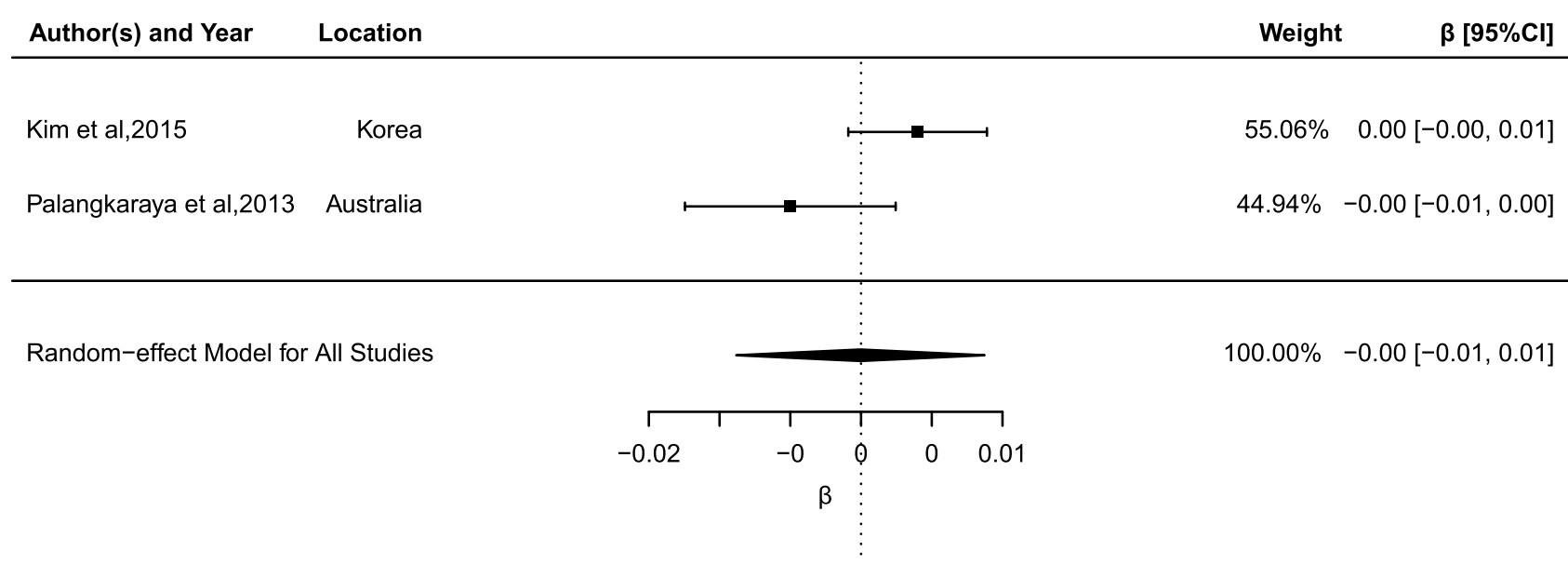

Figure 3 Forest plots of the effect of hospital competition on mortality.

Notes: The weight given to each study was the inverse of the SE of the effect estimate (inverse-variance method); $\beta>0$ implies higher HHI (lower degree of competition) is associated with higher mortality rate.

0.002). Concentrating on the same population, Kessler and McClellan ${ }^{41}$ found the 1-year mortality rate was higher in markets with very high $\mathrm{HHI}$ than those with very low $\mathrm{HHI}$ $(\beta=1.46$, SE 0.69), while there was no significant difference in markets with high $(\beta=0.46$, SE 0.57$)$ or low $(\beta=0.65$, SE 0.44$)$ HHI compared to markets with very low HHI. Chou et $\mathrm{al}^{44}$ showed that competition was negatively correlated with in-hospital mortality among patients who had undergone CABG surgery. They found the mortality rate for the very severely ill in the most competitive markets was approximately 0.0075 points lower, and for severely ill patients in competitive markets the reduction was 0.0127 points.Liao et $\mathrm{al}^{48}$ reported that competition and inpatient mortality were positively correlated among hemorrhagic (moderately vs highly competitive, $\beta=$ -0.0702 , SE 0.0135 ; less competitive vs highly competitive, $\beta=-0.0631$, SE 0.0107) and ischemic stroke patients (moderately vs highly competitive, $\beta=-0.0070$, SE 0.0023 ; less competitive vs highly competitive, $\beta=-0.0040$, SE 0.0019). The remaining study, Kessler and Geppert, ${ }^{43}$ showed that competition was not correlated with mortality rate for low-risk AMI patients (very high vs low HHI, $\beta=0.221$, SE 0.281,; high vs low HHI, $\beta=-0.083$, SE 0.197 ), while negatively correlated with mortality for high-risk AMI patients (very high vs low HHI, $\beta=0.822$, SE 0.319; high vs low HHI, $\beta=0.496$, SE 0.230).

\section{Secondary Outcome: Changes in LOS}

Only two studies reported on the impact of hospital competition on LOS, ${ }^{4,46}$ bothin South Korea. The first, ${ }^{45}$ on 279,847 patients presenting to hospital for joint-related diseases (included both surgical and nonsurgical procedures), suggested that LOS increased with lower competition $(\beta=0.023, P<0.0001)$. The second, which used data of 821,912 hospitalizations for hemorrhoids from 1,742 hospitals, on the contrary showed that LOS was higher in high-competition areas than moderate-competition areas $(\beta=0.01, P<0.0001)$, while no difference was found between low- and moderate-competition areas in terms of $\operatorname{LOS}(\beta=0.0002, P=0.9451){ }^{46}$

\section{Discussion}

All nine studies reported on the effect of competition on readmission, seven on mortality, and on LOS. Due to diverse forms of the HHI and different statistical models used, meta-analysis was not suitable for all of them.

One of the main purposes of competition among health-care providers is to attract patients and then increase their market share of medical services. The meta-analysis of three studies showed that competition may make no difference to unplanned readmissions. This may partially be due to readmissions being a providercentered quality indicator that is less sensitive than patient-centered quality indicators, such as satisfaction and waiting time. ${ }^{58}$ Because of the information asymmetry, patients usually do not have sufficient information about medical care quality. ${ }^{59}$ Therefore, disclosure of quality information may attract more patients.

The remaining studies reported inconsistent results, which may be due to different target diseases and a range of settings. Some researchers think that minor common diseases, such as hemorrhoids, may be more appropriate for competition-related research, because all 
hospitals can provide the required treatment and competition may be more evident. ${ }^{45,47}$ Studies focus on acute diseases (eg, heart attack) hold the opposite view: with severe conditions, patients may not have sufficient time to consider thoroughly which hospital to choose and are less willing to pay, and thus outcomes are in part a choice decision of the hospital itself. ${ }^{40,60}$ For settings, different countries have distinct health-care systems that vary in term of hospital privatization, reimbursement systems, payment methods, and generosity of insurance, so price and quality elasticity of demand are different as well. These also influence the effect of competition on quality: improving quality to attract patients or reducing quality to save costs. ${ }^{60}$ Therefore, when competition is used as a policy tool to improve quality of care, the health-care systemshould guide competition to produce the expected positive effects carefully. Besides, it is not clear whether measurement of market share, definition of hospital market, and varied outcome indicators (such as 30-day readmission, 1-year readmission) would have an impact on evaluating the effect of competition on readmissions. However, the number of eligible studies is too small to conduct subgroup analysis for controlling these biases. Consensus was unable to be reached from this review.

When taking readmission as proxy of hospital quality, mortality rate and LOS are the paired outcomes. However, some studies failed to report these. As such, the impact of competition on health-care quality needs further and more thorough investigation.

\section{Overall Completeness and Applicability of Evidence}

The primary outcome of readmission was reported in different formats: only three studies reported the most appropriate measure of estimator of the HHI $(\beta)$. The evidence for this pooled result is of low certainty, as we decided not to downgrade this for high risk of bias, as we began with low-certainty evidence when only observational studies were included. For the remaining six studies, usable data on this key outcome were limited and often unavailable. For the secondary outcomes, mortality was reported by seven studies; however, due to the lack of a clear methodology for the collection of partial HHI effects, only two studies were analyzed further. Only two studies reported LOS. The available evidence for all secondary outcomes is of very low certainty, because of indirectness and inconsistency.
The studies took place in a limited range of settings: the US, South Korea and Australia (high-income countries), and China (upper-middle-income country). There is a lack of evidence from relatively low-income countries. Target diseases of the studies varied a lot. Conclusions drawn from this review are likely to be directly relevant only to such diseases within such settings.

\section{Potential Biases in the Review Process}

Clearly described procedures were followed to prevent potential bias in the review process. We conducted a careful literature search, and the methods we used were transparent and reproducible. It is possible that potential studies published in journals that were outside our search strategy may have been missed. We attempted to contact two authors, but only one responded. Consequently, we may have underestimated the quality of some studies, simply because insufficient information prevented assessment of same. Additionally, this review only included studies published in English, thus may missed those published in other languages.

We looked for unplanned readmissions that were avoidable/preventable (through proper care delivery). However, no studies had identified whether the readmissions they explored were avoidable or not, and it was not possible for us to focus on avoidable readmissions only. In addition, none of thestudies discussed or controlled premises of associating readmissions with quality of care, and just two controlled the endogeneity of the HHI using predicted patient flow. All these factors can generate bias.

\section{Agreements and Disagreements with Other Studies or Reviews}

To the best of our knowledge, this is the only review to focus on the impact of hospital competition on readmissions. There have been three reviews evaluating the impact of competition on hospital quality from other perspectives. ${ }^{8,11,33}$ Gaynor et $\mathrm{al}^{8}$ reviewed all studies on hospital competition and quality published before 2011 without specifying competition or quality indicators. From other research (on 28 studies), two (included readmissions) $)^{41,43}$ overlapped with our review. This review narratively stated different outcome variables (readmission, mortality, and patient safety event) as a bundled quality-of-care outcome to explain the impact of competition on hospital quality. It did not reach any conclusion on the relationship between hospital competition 
and each quality indicator. Another review, Ghiasi et al, ${ }^{11}$ included only studies focusing on US hospitals with the aim of categorizing strategies and outcomes. Also, it reviewed the impact of competition on all these outcomes (eg, cost, mortality, and revenue) and listed 143 hospitalcompetition relationships. Among the65 studies included, only one ${ }^{44}$ used readmission as an outcome indicator, which was included in our review as well. Ghiasi et $\mathrm{al}^{11}$ briefly reported an inconsistency in the impact of competition on a number of dependent variables. Instead of mixing all the quality and competition indicators, the most recent review of hospital competition 33 included only studies that used 30-day mortality rate of AMI as a measure of health-care quality and the HHI as the measure of competition. It included eleven studies, and the meta-analysis showed that competition and mortality had a positive relationship, but not statistically significant. Our review concentrated on the impact of hospital competition on readmission and also reported its impact on mortality and LOS, as these were correlated with risk of readmission. Similar to Shen et $\mathrm{al}^{33}$ we included only studies measuring hospital competition with the HHI to reduce heterogeneity among studies.

It is also worth mentioning that our review is the only one in which GRADE assessment was used to highlight the certainty of the evidence.

\section{Conclusion}

\section{Implications for Policy-Makers}

The available evidence about the impact of competition on readmission is little: only nine studies were included, and it was impossible to pool all the results, due to the diverse methods used. Based on GRADE, we are uncertain whether or not competition has an effect on readmission rates, mortality, or LOS. However, evidence in this review should be carefully considered by health policy-makers for hospital-competition relevance, as there was a degree of heterogeneity in terms of different market environments.

\section{Implications for Research}

Many countries have introduced competition to their health-care systems. However, according to our investigation presented, it is clear that there is a serious lack of evaluation of the relationship between competition and readmissions. Therefore, current health-care policies are often based on unproven assumptions and may not guide the health-care system in the intended direction. More rigorous investigations are needed to clarify the effect of hospital competition and link policies to best health-care delivery by operational mechanisms, in order to facilitate benign competition.

First, it is essential to understand what exactly the context of hospital competition consists in. There are many vital questions remain to be answered What are the exact driving factors of competition in different settings (eg, private health-care providers vs public providers)? What really influences the choice of patients in different settings? What are the roles of regulation bodies in shaping the market that leads to competition?

Second, more comprehensive and precise tools for health-care quality and competition-strength evaluations need to be developed. The current tools assessing quality of care only cover one or two of the three aspects of medical quality (outcomes, processes, and structure). The widely used competition indicator, the HHI, troubles scholars by the endogeneity it generates, and again displayed its limitations in this review. Evaluation tools that can better reflect factors that affect hospital management and patient choice are important.

Finally, better methods of comparison across different health-care systems are required. This will be important to reveal how different policies and structures (eg, public vs private, price regulation, quantity regulation) influence the market and quality. In situations where prices are set administratively (eg, the UK National Health Service, and the Medicare program in the US), hospitals can only compete by quality of care. In other settings where the price of care is determined by the market, hospitals can attract patients not only through quality but also through lower expense. In this case, competition may not improve quality of care, as higher quality incurs higher costs. As such, different markets in different settings influence effects on competition and quality. As we revealed, even studies conducted in the same country showed conflicting results. Lack highquality original studies, this review failed to determine how health systems and country contexts influence the relationship between competition and quality of care. This is a complex but valuable aspect of investigating the competition problem that could provide plenty of clues and data.

It is of great importance to understand the impact of competition on hospital quality and then find the right way to guide competition to generate positive results. There is 
scope for future research in this area, as there is currently a lack of high-quality evidence. To make results comparable, we recommend that any future studies should:

- report the partial effect of the HHI with SE, especially when nonlinear regression is used

- report the result of the model where the HHI is included as a continuous variable, or use a standardized method to categorize the HHI if the author aims to investigate differences in effects for hospitals in high-, moderate-, and low-competition situations

- have an appropriate definition of readmissions with a time window after discharge, as if the time window is too short, patients may remain within the period during which hospitals provide follow-up care for their patients, while if it is too long the outcomes of patients may be caused by uncontrollable out-of-hospital factors, instead of hospital quality related to the initial admission.

\section{Funding}

This study was supported by the National Natural Science Foundation of China under grant 71874116, Ministry of Education of China under grant 18YJA790062, Chengdu Federation of Social Science Association under grant ZZ05, Sichuan University under grants 2018SCUH0027 and SKSYL201811, and China Medical Board under grant 17-276.

\section{Disclosure}

The authors report no potential conflicts of interest for this work.

\section{References}

1. Pan J, Qin X, Li Q, Messina JP, Delamater PL. Does hospital competition improve health care delivery in China? China Econ Rev 2015;33:179-199. doi:10.1016/j.chieco.2015.02.002

2. Longo F, Siciliani L, Moscelli G, Gravelle H. Does hospital competition improve efficiency? The effect of the patient choice reform in England. Health Econ. 2019;28(5):618-640. doi:10.1002/hec.3868

3. Frakt BA. Learning about competition from the UK's national health service. JAMA. 2015;314(6):547. doi:10.1001/jama.2015.9195

4. Porter ME, Teisberg EO. Redefining competition in health care. Harv Bus Rev. 2004;82(6):64-76, 136.

5. Brekke KR, Nuscheler R, Rune Straume O. Quality and location choices under price regulation. $J$ Econ Manag Strategy. 2006;15 (1):207-227. doi:10.1111/j.1530-9134.2006.00098.x

6. Teisberg EO, Porter ME, Brown GB. Making competition in health care work. Harv Bus Rev. 1994;72(4):131-141.

7. Blackstone EA, Fuhr JP. Redefining health care: creating value-based competition on results. Atl Econ J. 2007;35(4):491-501. doi:10.1007/ s11293-007-9091-9

8. Gaynor M, Town RJ. Chapter nine - competition in health care markets. In: Handbook of Health Economics. Vol. 2. 2011:499-637.
9. Dranove D. Chapter ten - health care markets, regulators, and certifiers. In: Pauly MV, Mcguire TG, Barros PP editors. Handbook of Health Economics. Vol. 2. Elsevier; 2011:639-690. doi:10.1016/ B978-0-444-53592-4.00010-4

10. Delamater PL, Messina JP, Grady SC, WinklerPrins V, Shortridge AM. Do more hospital beds lead to higher hospitalization rates? A spatial examination of Roemer's law. PLoS One. 2013;8(2):e54900. doi:10.1371/journal.pone.0054900

11. Ghiasi A, Zengul FD, Ozaydin Bet al. The impact of hospital competition on strategies and outcomes of hospitals: a systematic review of the US hospitals 1996-2016. J Health Care Finance. 2018;44(2).

12. Baker LC. Measuring competition in health care markets. Health Serv Res. 2001;36(1 Pt 2):223-251.

13. Chen -C-C, Cheng S-H. Hospital competition and patient-perceived quality of care: evidence from a single-payer system in Taiwan. Health Policy (New York). 2010;98(1):65-73. doi:10.1016/j.healthpol.2010.06.022

14. Pan J, Yan P, Liu M. Research progress on defining hospital markets from empirical perspective (in Chinese). Med Soc (Berkeley). 2016;29(8):75-77.

15. Wong HS, Zhan C, Mutter R. Do different measures of hospital competition matter in empirical investigations of hospital behavior. Rev Ind Organ. 2005;26(1):27-60. doi:10.1007/s11151-004-6067-7

16. Deffebach RR. Quality of care. JAMA. 1986;256(8):1032-1034. doi:10.1001/jama.1986.03380080078032

17. Corbett C, Daratha K, Setter S, Neumiller J Reducing acute care use by resolving medication discrepancies. In: 2013 Western Institute of Nursing Annual Communicating Nursing Research Conference; 2012.

18. Jencks SF, Williams MV, Coleman EA. Rehospitalizations among patients in the Medicare fee-for-service program. $N$ Engl $J$ Med. 2009;360(14):1418-1428. doi:10.1056/NEJMsa0803563

19. Khanna R, McDevitt JL, McClendon JJ, Smith ZA, Dahdaleh NS, Fessler RG. Utility of readmission rates as a quality of care measure and predictors of readmission within 30 days after spinal surgery: a single-center, multivariate analysis. Spine. 2015;40(22):1769-1774. doi:10.1097/BRS.0000000000001146

20. Lyons JS, O’Mahoney MT, Miller SI, Neme J, Kabat J, Miller F. Predicting readmission to the psychiatric hospital in a managed care environment: implications for quality indicators. Am J Psychiatry. 1997;154(3):337-340.

21. Courtney EDJ, Ankrett S, Mccollum PT. 28-Day emergency surgical re-admission rates as a clinical indicator of performance. Ann $R$ Coll Surg Engl. 2003;85(2):75-78. doi:10.1308/003588403321219803

22. Maurer PP, Ballmer PE. Hospital readmissions-Are they predictable and avoidable? Swiss Med Wkly. 2004;134(41-42):606-611.

23. Wadhera RK, Joynt Maddox KE, Wasfy JH, Haneuse S, Shen C, Yeh RW. Association of the hospital readmissions reduction program with mortality among medicare beneficiaries hospitalized for heart failure, acute myocardial infarction, and pneumonia. JAMA. 2018;320 (24):2542-2552. doi:10.1001/jama.2018.19232

24. De Francisco D, Anderson D, Pantano R, Kline F. The relationship between length of hospital stay and rapid-readmission rates. Hosp Community Psychiatry. 1980;31(3):196-197. doi:10.1176/ps.31.3. 196

25. Sud M, Yu B, Wijeysundera HC, et al. Associations between short or long length of stay and 30-day readmission and mortality in hospitalized patients with heart failure. JACC Heart Fail. 2017;5(8):578588. doi:10.1016/j.jchf.2017.03.012

26. Khan H, Greene SJ, Fonarow GC, et al. Length of hospital stay and 30-day readmission following heart failure hospitalization: insights from the EVEREST trial: length of hospital stay and 30-day readmissions. Eur J Heart Fail. 2015;17(10):1022-1031. doi:10.1002/ ejhf. 282

27. Sundaramurthi T. Predictors of Length of Hospital Stay and Readmission in Hematologic Stem Cell Transplant Recipients [Dissertations \& Theses Gradworks]. Published online 2015. 
28. Brekke KR, Siciliani L, Straume OR. Hospital competition and quality with regulated prices. Scand J Econ. 2011;113(2):444-469. doi:10.1111/j.1467-9442.2011.01647.x

29. Cooper Z, Gibbons S, Jones S, McGuire A. Does hospital competition save lives? Evidence from the English NHS patient choice reforms. Econ J. 2011;121(554):F228-F260. doi:10.1111/j.14680297.2011.02449.x

30. Gowrisankaran G, Town RJ. Competition, payers, and hospital quality. Health Serv Res. 2003;38(6p1):1403-1422. doi:10.1111/j.14756773.2003.00185.x

31. Mukamel DB, Zwanziger J, Tomaszewski K. HMO penetration, competition, and risk-adjusted hospital mortality. Health Serv Res. 2002;36(6 Pt 1):1019-1035.

32. Berta P, Martini G, Moscone F, Vittadini G. The association between asymmetric information, hospital competition and quality of healthcare: evidence from Italy. J R Stat Soc Ser A. 2016;179(4):907-926. doi: $10.1111 /$ rssa. 12214

33. Shen VC-Y, Ward WJ, Chen L-K. Systematic review and metaanalysis on the effect of hospital competition on quality of care: implications for senior care. Arch Gerontol Geriatr. 2019;83:263270. doi:10.1016/j.archger.2019.05.001

34. Downes MJ, Brennan ML, Williams HC, Dean RS. Development of a critical appraisal tool to assess the quality of cross-sectional studies (AXIS). BMJ Open. 2016;6(12):e011458. doi:10.1136/bmjopen2016-011458

35. Wong JN, McAuley E, Trinh L. Physical activity programming and counseling preferences among cancer survivors: a systematic review. Int J Behav Nutr Phys Act. 2018;15(1). doi:10.1186/s12966-0180680-6

36. Higgins JPT. Measuring inconsistency in meta-analyses. BMJ. 2003;327(7414):557-560. doi:10.1136/bmj.327.7414.557

37. Deeks JJ, Higgins JP, Altman DG. Chapter 10: analysing data and undertaking meta-analyses. In: Cochrane Handbook for Systematic Reviews of Interventions. 2019:241-284

38. Becker BJ, Wu M-J. The synthesis of regression slopes in metaanalysis. Stat Sci. 2007;22(3):414-429. doi:10.1214/07-STS243

39. Guyatt GH, Oxman AD, Kunz R, Vist GE, Falck-Ytter Y, Schünemann HJ. What is "quality of evidence" and why is it important to clinicians? BMJ. 2008;336(7651):995-998. doi:10.1136/ bmj.39490.551019.BE

40. Dunn A, Shapiro AH. Physician competition and the provision of care: evidence from heart attacks. Am J Health Econ. 2018;4(2):226261. doi:10.1162/ajhe a 00099

41. Kessler DP, McClellan MB. Is hospital competition socially wasteful? $Q J$ Econ. 2000;115(2):577-615. doi:10.1162/003355300554863

42. Ho V, Hamilton BH. Hospital mergers and acquisitions: does market consolidation harm patients? J Health Econ. 2000;19(5):767-791. doi:10.1016/S0167-6296(00)00052-7

43. Kessler DP, Geppert JJ. The effects of competition on variation in the quality and cost of medical care. J Econ Manag Strategy. 2005;14 (3):575-589. doi:10.1111/j.1530-9134.2005.00074.X

44. Chou S-Y, Deily ME, Li S, Lu Y. Competition and the impact of online hospital report cards. J Health Econ. 2014;34(3):42-58. doi:10.1016/j.jhealeco.2013.12.004

45. Kim SJ, Park E-C, Yoo K-B, Kwon JA, Kim TH. The association of market competition with hospital charges, length of stay, and quality outcomes for patients with joint diseases: a longitudinal study in Korea. Asia Pac J Public Health. 2015;27(2):195-207. doi:10.1177/ 1010539514529812
46. Kim SJ, Park E-C, Kim SJ, et al. The effect of competition on the relationship between the introduction of the DRG system and quality of care in Korea. Eur $J$ Public Health. 2016;26(1):42-47. doi:10.1093/eurpub/ckv162

47. Palangkaraya A, Yong J. Effects of competition on hospital quality: an examination using hospital administrative data. Eur $J$ Health Econ. 2013;14(3):415-429. doi:10.1007/s10198-012-0386-7

48. Liao C-H, Lu N, Tang C-H, Chang H-C, Huang K-C. Assessing the relationship between healthcare market competition and medical care quality under Taiwan's National Health Insurance programme. Eur J Public Health. 2018;28(6):1005-1011. doi:10.1093/eurpub/ cky099

49. Aggarwal AK, Sujenthiran A, Lewis D, et al. Impact of patient choice and hospital competition on patient outcomes after prostate cancer surgery: a national population-based study. Cancer. 2019;125 (11):1898-1907. doi:10.1002/cncr.31987

50. Hayford TB. The impact of hospital mergers on treatment intensity and health outcomes. Health Serv Res. 2012;47(3pt1):1008-1029. doi:10.1111/j.1475-6773.2011.01351.x

51. Mutter RL, Wong HS, Goldfarb MG. The effects of hospital competition on inpatient quality of care. Inquiry. 2008;45(3):263-279. doi:10.5034/inquiryjrnl_45.03.263

52. Siciliani L, Martin S. An empirical analysis of the impact of choice on waiting times. Health Econ. 2007;16(8):763-779. doi:10.1002/ hec. 1205

53. Chhatre S, Malkowicz SB, Schwartz JS, Jayadevappa R. Effects of hospital competition on racial ethnic disparity in prostate cancer care. J Urol. 2018;199(4):e564-e565. doi:10.1016/j.juro.2018.02.1369

54. Joynt K, Jha A. Does market competitiveness impact readmission rates for elderly patients with heart failure? Am Heart Assoc. 2010;122(suppl_21).

55. Lee $\mathrm{CW}, \mathrm{Wu} \mathrm{SC}$. The effect of market competition and risk factors on the utilization of acute psychiatric inpatients. Taiwan J Public Health. 2005;24:296-305.

56. Chua CL, Palangkaraya A, Yong J. Hospital competition, technical efficiency and quality. Econ Rec. 2011;87(277):252-268. doi:10.1111/j.1475-4932.2010.00704.x

57. Leleu H, Al-Amin M, Rosko M, et al. A robust analysis of hospital efficiency and factors affecting variability. Health Serv Manage Res. 2018;31(1):33-42. doi:10.1177/0951484817730537

58. Romano PS, Mutter R. The evolving science of quality measurement for hospitals: implications for studies of competition and consolidation. Int $J$ Health Care Finance Econ. 2004;4(2):131-157. doi:10.1023/B:IHFE.0000032420.18496.a4

59. Sofaer S, Firminger K. Patient perceptions of the quality of health services. Annu Rev Public Health. 2005;26:513-559. doi:10.1146/ annurev.publhealth.25.050503.153958

60. Propper C, Burgess S, Green K. Does competition between hospitals improve the quality of care? Hospital death rates and the NHS internal market. J Public Econ. 2004;88(7):1247-1272. doi:10.1016/ S0047-2727(02)00216-5

61. Liberati A, Altman D, Tetzlaff J, et al. The PRISMA statement for reporting systematic reviews and meta-analyses of studies that evaluate health care interventions: explanation and elaboration. Journal of clinical epidemiology. 2009;62(10). 


\section{Publish your work in this journal}

Risk Management and Healthcare Policy is an international, peerreviewed, open access journal focusing on all aspects of public health, policy, and preventative measures to promote good health and improve morbidity and mortality in the population. The journal welcomes submitted papers covering original research, basic science, clinical \& epidemiological studies, reviews and evaluations, guidelines, expert opinion and commentary, case reports and extended reports. The manuscript management system is completely online and includes a very quick and fair peer-review system, which is all easy to use. Visit http://www.dovepress.com/testimonials.php to read real quotes from published authors. 